\title{
Os impactos neurobiológicos e neurocognitivos da atividade física nos pacientes com esquizofrenia
}

The neurobiological and neurocognitive impacts of physical activity in patients with schizophrenia

Los impactos neurobiológicos y neurocognitivos de la actividad física en pacientes con esquizofrenia

Luís Felipe Malaquias Ferreira ${ }^{1 *}$, Ana Carolina Lobato Saldanha1 ${ }^{1}$, Ana Clara Teixeira Cherem ${ }^{1}$, Anna Lethícia dos Santos ${ }^{1}$, Ana Maria Martins de Freitas ${ }^{1}$, Marcos Vinícius Lino Campos ${ }^{1}$, Kelly Pereira Robis ${ }^{1,2}$.

\section{RESUMO}

Objetivo: Descrever o impacto neurobiológico, cognitivo e psicopatológico da atividade física nos indivíduos com esquizofrenia. Revisão Bibliográfica: $A$ evolução da esquizofrenia está relacionada às alterações neuropatológicas do sistema nervoso central, tanto em relação à estrutura, quanto em relação ao seu funcionamento. As alterações corticais refletem os aspectos clínicos da doença, como o comprometimento cognitivo, os sintomas positivos e negativos. A atividade física regular exerce um efeito neuroprotetor e neurogênico no cérebro, que parece ser capaz de retardar a neurodegeneração característica da doença com potencial para melhoria dos sintomas psicopatológicos e do desempenho cognitivo desses pacientes. Considerações Finais: Atividade física se mostrou benéfica em relação aos efeitos neurobiológicos, cognitivos e no controle de sintomas negativos. Todavia, não existem evidências da melhora do funcionamento psicossocial através da atividade física. Dessa forma há a necessidade de estudos com amostras maiores, envolvendo indivíduos em estágios iniciais ou amostras pré-clínicas, para avaliar de forma fidedigna o impacto da atividade física sobre o desempenho cognitivo e do funcionamento psicossocial.

Palavras chave: Esquizofrenia, Exercício físico, Tratamento.

\begin{abstract}
Objective: To describe the neurobiological, cognitive and psychopathological impact of physical activity on individuals with schizophrenia. Bibliographic Review: The evolution of schizophrenia is related to neuropathological changes in the central nervous system, both in relation to the structure, as well as in relation to its functioning. Cortical changes reflect the clinical aspects of the disease, such as cognitive impairment, positive and negative symptoms. Regular physical activity has a neuroprotective and neurogenic effect on the brain, which seems to be able to delay the neurodegeneration characteristic of the disease with the potential to improve the psychopathological symptoms and cognitive performance of these patients. Final Considerations: Physical activity proved to be beneficial in relation to the neurobiological, cognitive effects and in the control of negative symptoms. However, there is no evidence of an improvement in psychosocial functioning through physical activity. Thus, there is a need for studies with larger samples, involving individuals in early stages or preclinical samples, to reliably assess the impact of physical activity on cognitive performance and psychosocial functioning.
\end{abstract}

Keywords: Schizophrenia, Physical exercise, Treatment.

\footnotetext{
1 Pontifícia Universidade Católica de Minas Gerais (PUC - Minas), Belo Horizonte - MG.

*E-mail: luis.malaquias@sga.pucminas.br

2 Universidade Federal de Minas Gerais (UFMG), Belo Horizonte - MG.
} 
RESUMEN

Objetivo: Describir el impacto neurobiológico, cognitivo y psicopatológico de la actividad física en individuos con esquizofrenia. Revisión bibliográfica: La evolución de la esquizofrenia está relacionada con cambios neuropatológicos en el sistema nervioso central, ambos en relación con la estructura, así como en relación con su funcionamiento. Los cambios corticales reflejan los aspectos clínicos de la enfermedad, como el deterioro cognitivo, los síntomas positivos y negativos. La actividad física regular tiene un efecto neuroprotector y neurogénico en el cerebro, que parece ser capaz de retrasar la característica de neurodegeneración de la enfermedad con el potencial de mejorar los síntomas psicopatológicos y el rendimiento cognitivo de estos pacientes. Consideraciones Finales: Se ha demostrado que la actividad física es beneficiosa en relación con los efectos neurobiológicos y cognitivos y en el control de los síntomas negativos. Sin embargo, no hay evidencia de mejoría en el funcionamiento psicosocial a través de la actividad física. Por lo tanto, es necesario realizar estudios con muestras más grandes, que involucren a individuos en etapas tempranas o muestras preclínicas, para evaluar de manera confiable el impacto de la actividad física en el rendimiento cognitivo y el funcionamiento psicosocial.

Palabras clave: Esquizofrenia, Ejercicio físico, Terapia.

\section{INTRODUÇÃO}

A esquizofrenia é uma doença mental grave, que acomete 0,3 a $0,7 \%$ da população, segundo a quinta edição do Manual Diagnóstico e Estatístico de Transtornos Mentais (DSM-5) (APA, 2013). A doença apresenta um caráter crônico, podendo cursar com deterioração progressiva (KAPLAN H, et al., 2017). Os sintomas característicos envolvem disfunções cognitivas, comportamentais e emocionais, sendo que não há nenhum sinal ou sintoma que seja específico da doença (GORCZYNSKI P e FAULKNER G, 2010).

Com efeito, a esquizofrenia é uma síndrome clínica de apresentação variável, com alta heterogeneidade em relação às características clínicas e prognósticas entre indivíduos com a doença (APA, 2013). As pessoas com esse transtorno podem continuamente apresentar distorções da realidade, dos pensamentos e das percepções, além de prejuízos globais do funcionamento, sobretudo em contextos profissionais e sociais. (APA, 2013; CHAN V, 2017).

O prognóstico da esquizofrenia depende de um conjunto de fatores que incluem aspectos individuais, ambientais e biológicos (APA, 2013). Início precoce, prejuízo funcional pré-mórbido, história familiar positiva para esquizofrenia, uso de drogas e ser do sexo masculino estão associados ao pior desfecho (KAPLAN H, et al., 2017).

As características psicóticas da esquizofrenia costumam surgir entre o fim da adolescência e meados dos 30 anos, sendo que alterações da cognição, como prejuízos de aprendizado, e déficits das habilidades sociais podem frequentemente surgir desde a infância (BEREND M, et al., 2015). Mesmo com o tratamento medicamentoso, os prejuízos cognitivos podem persistir quando os sintomas da fase ativa da doença estão em remissão, contribuindo para a incapacidade relacionada à doença (ENGH JA, et al., 2015).

Os sintomas negativos têm um papel importante no prognóstico dos pacientes com esquizofrenia. As evidências científicas atuais apontam para tratamentos eficazes para os sintomas positivos da doença, tais como delírios, alucinações e desorganização do comportamento e da fala. No entanto, existem poucas evidências sobre intervenções que possam produzir melhorias dos sintomas negativos.

Estudos de estimulação cerebral, farmacológicos e relacionados a mudanças do estilo de vida sugerem possíveis estratégias para promover a melhora da funcionalidade dos pacientes com esquizofrenia, mas os estudos ainda são limitados (GORCZYNSKI P e FAULKNER G, 2010). A despeito dessa limitação, os estudos existentes apontam que intervenções não farmacológicas podem ser benéficas no controle dos sintomas, na preservação cognitiva e no funcionamento global. Dentre essas intervenções a prática regular de atividades físicas se destaca como uma modalidade terapêutica promissora no tratamento da esquizofrenia (GIRDLER SJ, et al., 2019). 
Tendo isso em vista, faz-se necessário considerar a referida intervenção como possibilidade terapêutica, sendo então definida como o movimento corporal produzido pelos músculos esqueléticos que requer gasto de energia acima do repouso (LUBANS D, et al., 2016). O exercício é uma forma de atividade física realizada com planejamento, como execução segundo um modelo estruturado, repetitivo, com o objetivo de manter ou promover melhorias de um ou mais elementos da aptidão física.

Os efeitos biológicos da atividade física regular sustentada no corpo humano incluem regulação cardiovascular, pulmonar, metabólica, musculoesquelética e imunológica, assim como alterações estruturais e funcionais do cérebro (FIRTH J, et al., 2017). Estudos anteriores demonstram que atividade física regular está associada a melhora do humor e do bem-estar em pacientes com esquizofrenia (MEENAKSHI D, et al., 2016; CHAN V, 2017).

Os mecanismos associados aos benefícios da atividade física para pessoas com alguma doença psiquiátrica são pouco conhecidos. A literatura sugere que os mecanismos plausíveis de mudança psicológica por meio de atividade física e exercício se enquadram em uma de três perspectivas amplas: (1) alterações bioquímicas, como aumento dos níveis de neurotransmissores (por exemplo, endorfinas ou serotonina); (2) alterações fisiológicas, como aumento da termogênese e melhora do desempenho cardiovascular e muscular; (3) mudanças psicológicas como maior autonomia, maior autoestima, fortalecimento do suporte social, aprimoramento da imagem pessoal e amplificação de sensações prazerosas (HO PA, et al., 2018; OERTELKNÖCHEL V, et al., 2014).

Com o objetivo de descrever o impacto biológico e clínico da atividade física nos indivíduos com esquizofrenia, reuniu-se nesta revisão pesquisas sobre as relações entre atividade física e esquizofrenia. $O$ presente estudo se concentrará nos seguintes aspectos: (1) Impacto morfológico e funcional da atividade física no sistema nervoso dos pacientes com esquizofrenia; (2) Impacto cognitivo e psicopatológico da atividade física nos pacientes com esquizofrenia. Por fim, serão discutidas novas perspectivas relacionadas à atividade física nos pacientes com esquizofrenia.

\section{REVISÃO BIBLIOGRÁFICA}

\section{Impacto morfológico e funcional da atividade física no sistema nervoso dos pacientes com esquizofrenia}

A evolução da esquizofrenia está relacionada às alterações neuropatológicas quantitativas do sistema nervoso central, tanto em relação à estrutura quanto em relação ao seu funcionamento (RAGLAND JD, et al., 2009). A esquizofrenia possui apresentação clínica e biológica heterogênea, portanto, não existem alterações cerebrais específicas da doença.

Tanto os sintomas negativos quanto os sintomas positivos da esquizofrenia estão associados a um provável funcionamento anormal de circuitos neurais que sustentam o funcionamento cognitivo (VANES LD, et al.,2019). O mecanismo associado ao surgimento dos sintomas não está claro, acredita-se que estas alterações podem estar associadas tanto ao desenvolvimento quanto à manutenção dos sintomas observados nos pacientes com esquizofrenia (SVATKOVA A, et al., 2015; VANES LD, et al.,2019).

$\mathrm{Na}$ esquizofrenia estão presentes anormalidades na morfologia cerebral, com redução generalizada da substância cinzenta, mas também de substância branca (HÖISTAD M, et al., 2009). Observa-se especificamente diminuição do volume do córtex temporal e pré-frontal e outras áreas subcorticais como hipocampo, amígdala e giro parahipocampal (SVATKOVA A, et al., 2015).

A esquizofrenia é atualmente considerada um transtorno da conectividade cerebral (ENGH JA, et al., 2015), caracterizado principalmente pelo acometimento da conexão entre o córtex pré-frontal e as áreas posteriores do cérebro. Estudos de neuroimagem funcional demonstram alterações da ativação de diversas vias neurais associadas ao controle do processamento cognitivo como córtex cingulado anterior (ACC) e córtex pré-frontal superior e lateral (PFC), córtex parietal e aspectos dos gânglios da base (VANES LD, et al., 2019). Ainda não está claro se essas alterações estruturais e funcionais são o resultado de uma alteração cerebral estável, ou se existe um processo de perda neuronal progressiva. 
Atualmente, é aceito pela literatura a presença de endofenótipos variáveis na esquizofrenia, sendo que o curso da doença parece heterogêneo entre os grupos cujas alterações cerebrais atróficas são relativamente estáveis ou progressivas (SVATKOVA A, et al., 2015).

A teoria vigente a respeito do neurodesenvolvimento da esquizofrenia sugere que fatores pré-natais, perinatais e ao longo da infância e adolescência contribuem para a fisiopatologia do transtorno. A doença ocorreria como resultado de um neurodesenvolvimento anormal iniciado ainda na fase fetal associado a fatores como: infecções, desnutrição materno-fetal, patologias placentárias e baixo peso ao nascer (GIRDLER SJ, et al., 2019).

Ao passo que durante a infância e adolescência, eventos relacionados a neuroplasticidade, sobretudo com a poda sináptica de circuitos celulares piramidadais no córtex pré-frontal, poderiam ter um influência importante na manifestação da esquizofrenia (GIRDLER SJ, et al., 2019).

As alterações neuropatológicas presentes na esquizofrenia podem ser secundárias às alterações da poda sináptica durante o desenvolvimento cerebral, que resultam, principalmente, na redução da atividade microglial e da neuroplasticidade. Essa hipótese é sustentada por estudos que demonstram a redução do fator neurotrófico derivado do cérebro (BDNF), um regulador da plasticidade sináptica, mediada pela microglia. A redução da plasticidade neuronal parece estar associada às alterações funcionais e morfológicas do sistema nervoso central dos indivíduos com esquizofrenia (DE PICKER LJ, et al., 2017).

Os estudos apontam que o exercício físico, por meio de uma cascata de processos celulares e moleculares relacionadas a neurogênese, aumenta plasticidade cerebral (DE LA ROSA A, et al., 2019; HÖTTING K e RÖDER B, 2013). O efeito pode reduzir a velocidade da neuroprogressão observada em pacientes com esquizofrenia, possivelmente mediada pelo aumento de BDNF (DE LA ROSA A, et al., 2019).

Uma meta-análise conduzida por Firth J et al. (2017) selecionou sete estudos que usaram a neuroimagem para examinar o impacto do exercício na estrutura e no funcionamento cerebral das pessoas com esquizofrenia.

A meta-análise evidenciou que o exercício físico pode aumentar o volume cerebral em pessoas com esquizofrenia. Embora as regiões que apresentaram aumento com atividade física teriam sido variáveis entre os estudos, o hipocampo foi a principal região envolvida, sugerindo melhora da memória de curto prazo.

Pajonk F et al. (2010), em um ensaio clínico randomizado, descreveu aumento significativo do volume do hipocampo nos pacientes com esquizofrenia submetidos a atividade física aeróbica três vezes por semana ao longo de 12 semanas $(p=0,01)$.

O estudo demonstrou que a mudança do volume hipocampal nesse grupo foi correlacionado a uma melhora no desempenho nos testes de memória de curto prazo $\left(r^{2}=0,51 ; P<0,05\right)$. No entanto, um estudo clínico anterior, não evidenciou aumento significativo do o volume de regiões específicas do cérebro dos pacientes com esquizofrenia ou controles saudáveis (SCHEEWE TW, et al., 2013).

Svatkova A et al. (2015) em um ensaio clínico randomizado envolvendo 33 pacientes portadores de esquizofrenia e 48 controles avaliou o efeito da atividade física aeróbica por 6 meses ( 40 minutos, duas vezes por semana) no volume e funcionamento cerebral.

O treinamento físico foi associado a melhora da conectividade cerebral de áreas envolvidas nas funções executivas e memória $(p<0,05)$, no entanto as alterações não foram significativamente associadas a melhora nas funções cognitivas com base em testes neuropsicológicos.

Os dados presentes na literatura sugerem que atividade física pode produzir aumento de estruturas cerebrais, sobretudo do hipocampo, e da conectividade cerebral nos pacientes com esquizofrenia (WOODWARD ML, et al., 2018; PAJONK F, et al., 2010; FIRTH J, et al., 2017). No entanto, os resultados sobre o efeito da atividade física na morfologia e funcionamento cerebral são heterogêneos.

Não está estabelecido que esse aumento da estrutura cerebral resulta em uma melhora nas funções cognitivas das pessoas com esquizofrenia, todavia, estudos sustentam a hipótese de um possível retardo da neuroprogressão da doença (SVATKOVA A, et al., 2015; MITTAL VA, et al., 2017). 
Estudos futuros que possibilitem o reconhecimento dos endofenótipos distintos da esquizofrenia assim como a separação dos grupos para a intervenção, podem esclarecer melhor o papel da atividade física no cérebro das pessoas com esquizofrenia.

\section{Impacto cognitivo e psicopatológico da atividade física nos pacientes com esquizofrenia}

A sintomatologia da esquizofrenia pode ser dividia em dois grupos, os sintomas positivos e os sintomas negativos (FISCHER BA e BUCHANAN RW, 2020). Os sintomas negativos estão associados a alterações de diversos domínios cognitivos como memória, funcionamento cognitivo, linguagem e funções executivas.

Considera-se na atualidade que essas anormalidades são inerentes ao próprio transtorno, e não são apenas o resultado dos sintomas psíquicos da fase ativa da doença ou das intervenções farmacológicas (ENGH JA, et al., 2015). O perfil neuropsicológico dos pacientes com esquizofrenia é heterogêneo, no entanto as alterações cognitivas podem estar presentes em 75\% indivíduos com esquizofrenia (BOSIA M, et al., 2018).

As alterações neurocognitivas estão relacionadas ao declínio do funcionamento social e profissional. Os mecanismos relacionados à psicopatologia da esquizofrenia são complexos. O surgimento dos sintomas positivos ocorrem principalmente em função da anormalidade de vias dopaminérgicas.

Acredita-se que o declínio cognitivo relacionado aos sintomas negativos ocorre em função das alterações das vias gabaérgicas e glutamatérgicas, mediadas por fatores inflamatórios e também por mudanças estruturais possivelmente relacionadas à síndrome metabólica (SOMMER I, et al., 2016; BOSIA M, et al., 2018).

A atividade física pode trazer benefícios no funcionamento cognitivo dos pacientes com esquizofrenia possivelmente relacionados ao aumento da expressão de genes relacionados a neuroplasticidade e à melhora do perfil metabólico (BOSIA M, et al., 2018).

Firth $\mathrm{J}$ et al. (2017) realizaram uma meta-análise que analisou 10 artigos, foram observados resultados neurocognitivos e de qualidade de vida em 385 participantes após 12 semanas de atividade física, 2 h/semana.

Os desfechos foram avaliados através do MCCB (MATRICS Consensus Cognitive Battery), ferramenta que possibilita analisar domínios cognitivos individuais com base em estudos analíticos de fatores da estrutura da cognição em psicóticos.

Esta especifica sete domínios individuais de cognição, que são: velocidade de processamento; atenção/vigilância; capacidade de aprendizado; raciocínio; resolução de problemas; cognição social e a memória (verbal, visual e de trabalho).

Chama a atenção neste estudo o fato de que as intervenções de exercícios supervisionadas por profissionais de atividade física (incluindo treinadores físicos e professores de yoga) melhoraram significativamente a cognição global $(N=6, n=197, g=0,47$, IC 95\% $=0,19-0,75, P<0,001, Q=2,44, I 2=$ $0 \%$ ) enquanto os supervisionados por outros profissionais (ou seja, apoio à saúde mental e equipe de pesquisa) não o fizeram ( $N=4, \mathrm{n}=186, \mathrm{~g}=0,2, \mathrm{IC} 95 \%=-0,09$ a $0,48, \mathrm{P}=0,5, \mathrm{Q}=1,81, \mathrm{I} 2=0 \%)$.

Em relação aos resultados cognitivos propriamente ditos, efeitos significativos do exercício foram observados em tarefas de "cognição social" ( $g=0,71$, IC $95 \%=0,27-1,15, P=0,002, n=81, \mathrm{I} 2=0 \%$ ) e atenção/vigilância ( $g=0,66$, IC 95\%=0,20-1,12, P=0,005, $\mathrm{n}=104, \mathrm{I} 2=20,3 \%$ ).

O domínio cognitivo mais amplamente avaliado foi memória de trabalho $(\mathrm{N}=7, \mathrm{n}=282)$, no qual o exercício resultou em melhorias significativas em relação às condições de controle $(g=0,39, I C 95 \%=0,05-0,73, P=$ $0,024)$ com heterogeneidade média $(Q=10,92, P=0,091, I 2=45,1 \%)$. Os restantes dos domínios cognitivos individuais não apresentaram melhora significativa (FIRTH J, et al., 2017).

A revisão sistemática feita por Gorczynski P e Faulkner G (2010), analisou 442 publicações acerca da relação entre exercício físico e esquizofrenia, destas foram incluídos três estudos controle randomizados. Concluiu-se que a prática de exercícios físicos é capaz de melhorar os sintomas negativos da esquizofrenia de forma significativa (MD 17.50 IC 6.70 para 28.30, PANSS negativo IC -11.11 para -5.89 ), todavia, nenhuma melhora significante foi observada nos sintomas positivos da doença. 
Silva BA et al. (2015) realizaram um ensaio clínico randomizado avaliou o efeito do exercício físico em 50 pacientes homens com esquizofrenia de 18 a 50 anos com tratamento medicamentoso estável. Os pacientes foram divididos em três grupos: controle $(n=13)$, exercício de resistência (RESEX) $(n=12)$ e exercício concorrente (CONCEX) $(n=9)$. Os participantes deveriam praticar exercícios físicos duas vezes na semana durante 60 minutos por vinte semanas. $O$ grupo que realizou o exercício de resistência obteve melhores resultados.

A avaliação na décima semana mostrou melhora nos sintomas positivos apenas no RESEX com a diferença $(p=0.039)$ na média variável de interesse entre os grupos que não apresentaram nenhuma melhora. Já na vigésima semana, a melhora de RESEX em relação à média ( $\leq 0.001)$, enquanto no grupo CONCEX a melhora dos sintomas positivos apareceu pela primeira vez com a diferença $(p=0.016)$ quando comparado com os outros dois grupos, o grupo controle não apresentou alterações.

Quanto aos sintomas negativos, observou-se melhora em relação a interação de tempo por grupo com diferença significativa $(p=0.027)$ na média variável, especificamente no grupo RESEX que apresentou melhora tanto na décima semana $(p=0.001)$ e na vigésima semana $(p=0.002)$.

O estudo concluiu que o exercício físico pode ser uma estratégia não farmacológica para o tratamento de pacientes esquizofrênicos com a melhora dos sintomas positivos e negativos quanto associada ao uso dos medicamentos. Além de melhora na qualidade de vida, avaliada utilizando o SF-36 (SILVA BA, et al., 2015).

Dauwan e colaboradores, em 2016, conduziram uma revisão sistemática seguida de meta-análise analisando ensaios clínicos que investigaram os efeitos da prática de atividade física sobre os sintomas clínicos, qualidade de vida, funcionamento global e cognição em pacientes com transtornos do espectro da esquizofrenia. A amostra total foi de 1109 pacientes envolvidos em 29 estudos.

O exercício foi superior às condições de controle na melhoria da gravidade total dos sintomas $(\mathrm{k}=14, \mathrm{n}=$ 719: Hedges ' $g=.39, P<.001)$, positivo $(k=15, n=715$ : Hedges' $g=.32, P<0,01)$, negativo $(k=18, n=854$ : Hedges ' $g=0,49, P<0,001)$ e geral $(k=10, n=475$ : Hedges' $g=0,27, P<.05)$ sintomas, qualidade de vida $(\mathrm{k}=11, \mathrm{n}=770$ : Hedges ' $\mathrm{g}=.55, \mathrm{P}<.001)$, funcionamento global $(\mathrm{k}=5, \mathrm{n}=342$ : Hedges' $\mathrm{g}=.32, \mathrm{P}<.01)$, e sintomas depressivos ( $k=7, n=337$ : Hedges ' $g=0,71, P<0,001)$ (DAUWAN M, et al., 2016).

Bernard P e Ninot G (2012) conduziram uma revisão sistemática avaliando os benefícios do exercício físico para pacientes com esquizofrenia nos bancos de dados psycINFO, Medline / PubMed, SportDiscus, Web of Sciences e Biblioteca Cochrane.

Dos 139 artigo encontrados, 19 se adequaram aos critérios e em quinze deles houve associação estástica significativa $(p<0,05)$ entre a melhora de sintomas positivos e negativos. Porém, algumas limitações foram observadas como a ausência de estudos de longo prazo, amostras pequenas e heterogêneas (BERNARD $P$ e NINOT G, 2012).

Os estudos de revisão presentes na literatura evidenciam que a prática de exercício físico regular é capaz de alterar positivamente os sintomas negativos da esquizofrenia e dos domínios cognitivos, principalmente da memória de trabalho (FIRTH J, et al., 2017; GORCZYNSKI P e FAULKNER G, 2010; ROSENBAUM S, et al., 2014). No entanto, não existem evidências de que essas melhoras cognitivas resultam em melhora no funcionamento psicossocial das pessoas com esquizofrenia (DE SILVA MJ, et al., 2013).

As alterações das vias glutamatérgicas e gabaérgicas parecem estar presentes mesmo antes do surgimento dos sintomas psicóticos, o que pode refletir o início precoce de um declínio progressivo da cognição (SOMMER I, et al., 2016).

Considerando o início precoce do comprometimento cognitivo e a ausência da reversão desse processo por meio do exercício físico em estágios intermediários e avançados da doença, é necessária a formulação de estratégias de identificação de indivíduos de alto risco para o desenvolvimento da esquizofrenia e a criação de estratégias preventivas que possam promover o retardo da progressão dos quadros. 


\section{CONSIDERAÇÕES FINAIS}

A partir da análise dos artigos reunidos nesta revisão fica evidente que a esquizofrenia é uma doença complexa em seus diversas aspectos, desde a etiopatogenia e fisiopatologia, passando por suas manifestações clínicas e neuro-morfológicas até o seu tratamento. As evidências analisadas indicam a existência de uma possível neurodegeneração fronto-parietal correlacionada à deterioração cognitiva observada ao longo da progressão da doença. Os estudos mostraram que a atividade física contribui para a melhora do funcionamento cognitivo e para o aumento de diversas áreas cerebrais possivelmente relacionado ao aumento da neuroplasticidade. Ensaios com amostras maiores e com maior tempo de acompanhamento, com a separação de grupos com características específicos da progressão da doença, são necessários para a compreensão dos mecanismos relacionados ao efeito da atividade física na cognição. Apesar das evidências relacionadas a melhora da cognição dos pacientes submetidos a atividade física, não existem evidências de que essa melhora resulta em um desfecho psicossocial favorável. Uma possível justificativa para esse fenômeno é a existência de um declínio cognitivo prévio que não é revertido pela atividade física, que revela a necessidade de intervenções preventivas em amostras pré-clínicas.

\section{REFERÊNCIAS}

1. AMERICAN PSYCHIATRIC ASSOCIATION. Diagnostic and statistical manual of mental disorders. 5nd ed. Arlington, VA: American Psychiatric Publishing. Washington, DC, 2013; 72-76p.

2. BEREND $M$, et al. Effects of endurance training combined with cognitive remediation on everyday functioning, symptoms, and cognition in multiepisode schizophrenia patients. Schizophr Bull, 2015;41(4):847-858.

3. BERNARD P, NINOT G. Bénéfices des activités physiques adaptées dans la prise en charge de la schizophrénie : revue systématique de la littérature [Benefits of exercise for people with schizophrenia: a systematic review]. Encephale, 2012; 38(4):280-7.

4. BOSIA M, et al. Improving Cognition to Increase Treatment Efficacy in Schizophrenia: Effects of Metabolic Syndrome on Cognitive Remediation's Outcome. Front Psychiatry, 2018;9:647.

5. CAREK PJ, et al. Exercise for the treatment of depression and anxiety. Int J Psychiatry Med, 2011;41(1):15-28.

6. CASPERSEN CJ, et al. Physical activity, exercise, and physical fitness: definitions and distinctions for health-related research. Public Health Rep, 1985;100(2):126-131.

7. CHAN V. Schizophrenia and Psychosis: Diagnosis, Current Research Trends, and Model Treatment Approaches with Implications for Transitional Age Youth. Child and Adolescent Psychiatric Clinics of North America, 2017; 26(2):341 366

8. CURCIC D, et al. Positive impact of prescribed physical activity on symptoms of schizophrenia: randomized clinical trial. Psychiatr Danub, 2017;29(4):459-465.

9. DAUWAN M, et al. Exercise Improves Clinical Symptoms, Quality of Life, Global Functioning, and Depression in Schizophrenia: A Systematic Review and Meta-analysis. Schizophrenia Bulletin, 2016; 42(3):588-99.

10. DE LA ROSA A, et al. Long-term exercise training improves memory in middle-aged men and modulates peripheral levels of BDNF and Cathepsin B. Sci Rep, 2019; 9: 3337.

11. DE PICKER LJ, et al. Microglia and Brain Plasticity in Acute Psychosis and Schizophrenia Illness Course: A MetaReview. Frontiers in Psychiatry, 2017; 8:238.

12. DE SILVA MJ, et al. Effect of psychosocial interventions on social functioning in depression and schizophrenia: metaanalysis. Br J Psychiatry, 2013;202(4):253-260.

13. ENGH JA, et al. Effects of high-intensity aerobic exercise in psychotic symptoms and neurocognition in outpatients with schizophrenia: study protocol or randomized controlled trial. Trials, 2015; 16:557.

14. FIRTH J, et al. Aerobic exercise improves cognitive functioning in people with schizophrenia: A systematic review and meta-analysis. Schizophr Bull, 2017;43(3):546-556.

15. FIRTH J, et al. The pro-cognitive mechanisms of physical exercise in people with schizophrenia. Br J Pharmacol, 2017;174(19):3161-3172.

16. FISCHER BA, BUCHANAN RW. Schizophrenia in adults: clinical manifestations, course, assessment, and diagnosis. Uptodate, 2020.

17. GIRDLE SJ, et al. Exercício como tratamento para esquizofrenia: uma revisão. Psychopharmacology Bulletin, 2019; 49 (1): 56-69.

18. GORCZYNSKI P, FAULKNER G.Exercise therapy for schizophrenia. Cochrane Database Syst Rev, 2010;(5):CD004412.

19. HO PA, et al. Why do people with schizophrenia exercise? A mixed methods analysis among community dwelling regular exercisers. Front Psychiatry, 2018;9:596.

20. HÖISTAD M, et al. Linking white and grey matter in schizophrenia: Oligodendrocyte and neuron pathology in the prefrontal cortex. Frontiers in Neuroanatomy, 2009;3:9.

21. HÖTTING K, RÖDER B. Beneficial effects of physical exercise on neuroplasticity and cognition. Neuroscience \& Biobehavioral Reviews, 2013; 37(9B): 2243-2257. 
22. KANDOLA A, et al. Moving to beat anxiety: Epidemiology and therapeutic issues with physical activity for anxiety. Curr Psychiatry Rep, 2018;20(8):63.

23. KAPLAN H, SADOCK B, GREBB J. Compêndio de Psiquiatria: ciência, comportamento e psiquiatria clínica. 11th ed. Porto Alegre: Artmed, 2017.

24. KIMHY D, et al. Aerobic fitness and body mass index in individuals with schizophrenia: Implications for neurocognition and daily functioning. Psychiatry Research, 2014; 220(3):784-791.

25. LINDAMER LA, et al. Assessment of physical activity in middle-aged and older adults with schizophrenia. Schizophr Res, 2008;104:294-301.

26. LUBANS D. Physical Activity for Cognitive and Mental Health in Youth: A Systematic Review of Mechanisms. Pediatrics, 2016;138(3):e20161642.

27. MEENAKSHI D, et al. Exercise improves clinical symptoms, quality of life, global functioning, and depression in schizophrenia: A systematic review and meta-analysis. Schizophr Bull, 2016;42(3):588-599.

28. MITTAL VA, et al. Exercise treatments for psychosis: a review. Curr Treat Options Psych, 2017; 4:152-166.

29. NASCIMENTO MIC, et al. Manual diagnóstico e estatístico de transtornos mentais: DSM-5. 5th ed. Porto Alegre: Artmed,2014.

30. OERTEL-KNÖCHEL V, et al. Effects of aerobic exercise on cognitive performance and individual psychopathology in depressive and schizophrenia patients. Eur Arch Psychiatry Clin Neurosci, 2014;264:589-604.

31. PAJONK F, et al. Hippocampal plasticity in response to exercise in schizophrenia. Arch Gen Psychiatry, 2010;67(2):133-143.

32. RAGLAND JD, et al. Prefrontal activation deficits during episodic memory in schizophrenia. American Journal of Psychiatry, 2009;166(8):863-874.

33. ROSENBAUM S, et al. Physical activity interventions for people with mental illness: a systematic review and metaanalysis. The Journal of Clinical Psychiatry, 2014; 75(9):964-974.

34. SCHEEWE TW, et al. Exercise therapy, cardiorespiratory fitness and their effect on brain volumes: a randomised controlled trial in patients with schizophrenia and healthy controls. Eur Neuropsychopharmacol, 2013;23(7):675-685.

35. SILVA BA, et al. A 20-week program of resistance or concurrent exercise improves symptoms of schizophrenia: results of a blind, randomized controlled trial. Braz J Psychiatry, 2015; 37(4):271-279.

36. SOMMER I, et al. Early interventions in risk groups for schizophrenia: what are we waiting for?. Npj Schizophr, 2016;2:16003.

37. SVATKOVA A, et al. Physical exercise keeps the brain connected: Biking increases white matter integrity in patients with schizophrenia and healthy controls. Schizophr Bull, 2015;41(4):869-878.

38. VANES LD, et al. Neural correlates of positive and negative symptoms through the illness course: an fMRI study in early psychosis and chronic schizophrenia. Sci Rep, 2019; 9:3337.

39. WEGNER $M$, et al. Effects of exercise on anxiety and depression disorders: review of meta- analyses and neurobiological mechanisms. CNS Neurol Disord Drug Targets, 2014;13(6):1002-1014.

40. WOODWARD, ML, et al. Hippocampal volume and vasculature before and after exercise in treatment-resistant schizophrenia. Schizophr Res, 2018; 202: 158-165. 